\title{
Contractility Dominates Adhesive Ligand Density in Regulating Cellular De-adhesion and Retraction Kinetics
}

\author{
Shamik Sen, ${ }^{1,3}$ Win Pin NG, ${ }^{2}$ and Sanjay Kumar ${ }^{1,2}$ \\ ${ }^{1}$ Department of Bioengineering, University of California, Berkeley, CA 94720-1762, USA; ${ }^{2}$ UCSF-UC Berkeley Joint Graduate \\ Group in Bioengineering, Berkeley, CA, USA; and ${ }^{3}$ Present Address: Department of Biosciences and Bioengineering, \\ Indian Institute of Technology Bombay, Mumbai, India
}

(Received 31 August 2010; accepted 17 October 2010; published online 29 October 2010)

Associate Editor Angelique Louie oversaw the review of this article.

\begin{abstract}
Cells that are enzymatically detached from a solid substrate rapidly round up as the tensile prestress in the cytoskeleton is suddenly unopposed by cell-ECM adhesions. We recently showed that this retraction follows sigmoidal kinetics with time constants that correlate closely with cortical stiffness values. This raises the promising prospect that these de-adhesion measurements may be used for highthroughput screening of cell mechanical properties; however, an important limitation to doing so is the possibility that the retraction kinetics may also be influenced and potentially rate-limited by the time needed to sever matrix adhesions. In this study, we address this open question by separating contributions of contractility and adhesion to cellular de-adhesion and retraction kinetics. We first develop serum-free conditions under which U373 MG glioma cells can be cultured on substrates of fixed fibronectin density without direct matrix contributions from the medium. We show that while spreading area increases with ECM protein density, cortical stiffness and the time constants of retraction do not. Conversely, addition of lysophosphatidic acid (LPA) to stimulate cell contractility strongly speeds retraction, independent of the initial matrix protein density and LPA's contributions to spreading area. All of these trends hold in serum-rich medium commonly used in tissue culture, with the time constants of retraction much more closely tracking cortical stiffness than adhesive ligand density or cell spreading. These results support the use of cellular de-adhesion measurements to track cellular mechanical properties.
\end{abstract}

Keywords-Adhesion, Contractility, Extracellular matrix (ECM), Ligand density, Serum, Myosin, Cell mechanics, Atomic force microscopy, Cell stiffness.

\section{ABBREVIATIONS}

GBM Glioblastoma Multiforme

ECM Extracellular matrix

Address correspondence to Sanjay Kumar, Department of Bioengineering, University of California, Berkeley, CA 94720-1762, USA. Electronic mail: skumar@berkeley.edu
NMMII Non-muscle myosin II

pMLC Phosphorylated myosin light chain

\section{INTRODUCTION}

Cells adherent to planar extracellular matrix (ECM) substrates exist in a force balance in which tensile forces in the cytoskeleton are opposed by the mechanical rigidity of the ECM. This "tensional homeostasis" plays key regulatory roles in a wide variety of cellular phenomena, including shape determination, migration, tissue assembly, and fate choice. ${ }^{3,6,27}$ Moreover, there is growing evidence that changes in cellular mechanical properties relevant to this force balance may serve as biomarkers for disease, including various types of cancer. ${ }^{17,24}$ Many methods have been introduced to measure single-cell mechanical properties, including atomic force microscopy, ${ }^{4,5}$ micropipette aspiration, ${ }^{10,15}$ traction force microscopy, ${ }^{22,28}$ optical tweezers, ${ }^{14,32,33}$ and intracellular particle tracking. ${ }^{8,31}$ While all of these methods have proven extremely powerful in producing high-resolution measurements of cellular viscoelastic properties, efforts to incorporate these methods into highthroughput technologies that might be used to diagnose disease or screen drug libraries based on changes in cell mechanics have been limited in part by these methods' inherently low throughput and low proclivity toward automation. This in turn has spawned a new generation of high-throughput cell mechanics technologies including optical stretchers ${ }^{7,12}$ and controlled detachment assays. ${ }^{30}$

We recently introduced an assay in which cellular tensional homeostasis could be gauged by tracking the kinetics with which cells retract following chemical detachment from the ECM. ${ }^{21}$ In this assay, cells are 
treated with trypsin to rapidly sever cell-ECM contacts and time-lapse phase-contrast imaging is used to track the projected cell area as a function of time. The idea is that as cell-ECM contacts are severed, tensile loads within the cytoskeleton suddenly become unbalanced, causing the cell to rapidly contract to a rounded morphology. We showed that the kinetics of this retraction obeys sigmoidal kinetics with characteristic time constants, and that the magnitude of these time constants closely track cellular elasticity as measured by AFM. The time constants also change in predictable ways when intracellular tension is either increased or decreased by pharmacologic inhibition or activation of cell contractility. An important unresolved issue with this assay, and with controlled-detachment assays in general, is that it is not clear to what extent cellECM adhesion itself contributes to the kinetics of the detachment process. In an extreme case, one might imagine that the rate of retraction could be limited by the time needed to dissipate cell-ECM contacts rather than relaxation of tensile forces within the cytoskeleton and, therefore, primarily reflect the strength of adhesion to the matrix. Distinguishing between contributions from these two sources is critical if this technology is to be explored for high-throughput screening of cell mechanical properties.

In this study, we address this open question by experimentally distinguishing contributions of cellular prestress and cell-ECM adhesion to retraction kinetics following de-adhesion. We culture cells on ECM substrates coated with varying densities of fibronectin and in the presence or in the absence of lysophosphatidic acid (LPA) to stimulate contractility. Critically, we conduct these studies in serum-free medium to minimize contributions of serum to matrix density and baseline contractility. We then perform de-adhesion assays and show that the time constants of retraction are relatively insensitive to changes in matrix density but are highly sensitive to perturbations in cell contractility. These conclusions are supported by immunofluorescence localization of key adhesive and contractile markers and by AFM nanoindentation measurements of cortical stiffness. Together, our data support the notion that cellular retraction kinetics following de-adhesion is dominated by the tensile state of the cell rather than the extent of cell-ECM adhesive engagement.

\section{MATERIALS AND METHODS}

\section{Cell Culture}

U373 MG human glioblastoma cells (American Type Culture Collection, MD) were cultured at $37{ }^{\circ} \mathrm{C}$ in a humidified atmosphere of $95 \%$ air and $5 \% \mathrm{CO}_{2}$ in growth medium composed of DMEM supplemented with $10 \%$ fetal calf serum, $1 \%$ sodium pyruvate (Invitrogen), 1\% non-essential amino acids (Invitrogen), and $1 \%$ penicillin/streptomycin (Invitrogen). Cells were maintained in $75 \mathrm{~cm}^{2}$ cell culture flasks (Corning, NY), harvested with $0.25 \%$ trypsin-EDTA (Invitrogen), and passaged every 2-3 days. For experiments, cells were plated on glass coverslips coated with human plasma fibronectin (Milipore Corp.) at various dilutions to achieve nominal (theoretical) coating densities of $0.1,1.0$, or $10.0 \mu \mathrm{g} / \mathrm{cm}^{2}$, respectively. Experiments were performed either in $10 \%$ serum-containing medium as described above or in serum-free medium (DMEM supplemented with 1\% sodium pyruvate, $1 \%$ non-essential amino acids, and $1 \%$ penicillin/streptomycin).

\section{Immunofluorescence Labeling and Antibodies}

Cultured cells were rinsed twice with phosphatebuffered saline (PBS) (Fisher Scientific), fixed with 4\% para-formaldehyde solution for $10 \mathrm{~min}$, permeabilized with $0.5 \%$ Triton $\mathrm{X}-100$, and blocked in $5 \%$ bovine serum albumin for $1 \mathrm{~h}$ at room temperature. Cells were incubated for $1 \mathrm{~h}$ at room temperature in one of the following primary antibody solutions in PBS at the following dilutions: mouse anti-vinculin (1:200, Sigma), rabbit anti-NMMII (1:200 anti-NMMIIA and 1:200 anti-NMMIIB, Sigma), and phosphorylated MLC (pMLC) (1:500, Cell Signaling). After incubation with primary antibody, cells were rinsed twice with PBS and then incubated with secondary antibody (either Alexa 543 goat anti-mouse IgG (1:500, Invitrogen) or Alexa 543 goat anti-rabbit $\operatorname{IgG}$ (1:500, Invitrogen)), and Alexa 488-phalloidin (1:200, Invitrogen) for $1 \mathrm{~h}$ at room temperature. Samples were mounted onto slides using Cytoseal 60 (Richard-Allan Scientific).

\section{Measurement of ECM Coating}

The fibronectin coating of glass coverslips at the different theoretical densities was quantified using immunofluorescence and Western blots. For quantitative immunofluorescence analysis, camera exposure and gain settings were fixed, and images were taken at 10 random positions for each condition. Median intensities of the histograms from each image were combined to determine the intensity distributions arising from surface-attached fibronectin across the different conditions. For Western blots, the total surface-adsorbed fibronectin for each condition was extracted by incubating fibronectin-coated surfaces with RIPA buffer for 5 min at room temperature and subsequently scraping with a cell scraper. The total amounts of collected protein corresponding to the three coating densities were boiled, run on a $4-12 \%$ 
Bis-Tris gel (Invitrogen), transferred to a PVDF membrane (Invitrogen), and probed with mouse monoclonal fibronectin antibody (1:500, Sigma). The blot was then probed with HRP-conjugated goat-antimouse (Invitrogen) and developed using TMB chromogenic substrates (Invitrogen). The developed blot was scanned, and the band intensities were quantified using Image $\mathbf{J}(\mathrm{NIH})$ software.

\section{Image Acquisition and Analysis}

Live-cell and epifluorescence images were obtained with a Nikon TE2000E2 microscope equipped with an incubator chamber for controlled temperature, humidity, and $\mathrm{CO}_{2}$ (In Vivo Scientific). Images were captured with a CCD camera (Photometrics CoolSNAP HQ2) integrated with image acquisition software (Compix SimplePCI). De-adhesion measurements were acquired as described previously. ${ }^{21}$ Briefly, the medium was aspirated off, cells were washed with PBS and incubated with warm trypsin, and images were acquired every $2 \mathrm{~s}$ at $20 \times$ magnification until cells became rounded while still remaining attached to the substrate. De-adhesion dynamics were quantified by fitting the plot of the timedependent normalized area to a sigmoidal curve with two time constants $\left(\tau_{1}\right.$ and $\left.\tau_{2}\right)$.

\section{Atomic Force Microscopy (AFM)}

Force curves were obtained with an Asylum MFP3D AFM (Asylum Research, CA) mounted on a Nikon TE2000U epifluorescence microscope. Cells were cultured overnight on glass coverslips, and 50-100 cells were indented for each condition using a pyramid-tipped probe (Veeco Instruments). Tips with nominal spring constant of $60 \mathrm{pN} / \mathrm{nm}$ were calibrated using the thermal noise method, and the calibrated values were used for all calculations. The elastic modulus of the cell was determined by fitting each force curve with a modified Hertzian model of a cone indenting a semi-infinite elastic solid. ${ }^{20}$

\section{RESULTS}

\section{Quantification of Fibronectin Surface Adsorption}

To systematically probe contributions of ECM ligand density to cellular de-adhesion and retraction kinetics, we created ECM substrates of varying fibronectin density by functionalizing glass coverslips at three theoretical coating densities, $0.1,1.0$, and $10 \mu \mathrm{g} /$ $\mathrm{cm}^{2}$. We then characterized the degree of surface coverage in each case by immunofluorescence imaging (Fig. 1a). Quantitative analysis of total surface fluorescence revealed a tenfold increase in intensity levels from 0.1 to $1.0 \mu \mathrm{g} / \mathrm{cm}^{2}$, with no further increase in intensity from 1.0 to $10 \mu \mathrm{g} / \mathrm{cm}^{2}$, implying saturation of fibronectin adsorption or detection under these conditions (Fig. 1b). Quantification of immunofluorescence images is often complicated by sample-tosample variations in photobleaching, illumination intensity, and other parameters. Thus, we further characterized adsorption densities by harvesting surface-adsorbed fibronectin and subjecting this lysate to SDS-PAGE followed by Western blot against fibronectin (Fig. 1c). As expected, we observed multiple bands scattered across $200-250 \mathrm{kDa}$, the expected molecular weight range for fibronectin fragments. ${ }^{11}$ Subsequent densitometric analysis of the band intensities revealed a fivefold increase in intensity between a theoretical $1 \mu \mathrm{g} / \mathrm{cm}^{2}$ surface and a $0.1 \mu \mathrm{g} / \mathrm{cm}^{2}$ surface, and a much smaller increase in intensity between $1 \mu \mathrm{g} / \mathrm{cm}^{2}$ and $10 \mu \mathrm{g} / \mathrm{cm}^{2}$.

\section{Fibronectin Density-Dependent Adhesion Under Serum-Free Conditions}

We next used these ECM substrates to investigate the relationship between surface ECM density and cell spreading and retraction kinetics following de-adhesion. To simultaneously eliminate contributions of fibronectin adsorption from the medium and minimize intrinsic levels of cell contractility, we chose to work with serum-free medium and then add lysophosphatidic acid (LPA) to specifically stimulate Rho GTPasedependent cell contractility. ${ }^{13}$ To quantify the effect of ECM density on cell spreading area under serum-free conditions, we cultured equal numbers of U373 MG glioma cells overnight on fibronectin-coated glass coverslips at specific coating densities. Phase contrast imaging and subsequent morphometric analysis (Fig. 2a, top panel; Fig. 2b, left side) demonstrated that the spreading of these cells is sensitive to changes in ECM density. First, as expected, fewer cells adhered to $0.1 \mu \mathrm{g} / \mathrm{cm}^{2}$ substrates than to 1.0 and $10 \mu \mathrm{g} / \mathrm{cm}^{2}$ substrates. Second, although cells exhibited a spindlelike shape on all substrates, their projected spreading area increased with increasing fibronectin density. Consistent with our observation that fibronectin adsorption saturates above $1.0 \mu \mathrm{g} / \mathrm{cm}^{2}$ (Fig. 1), cell spreading increased significantly (blue bars, $* p<0.05$ ) from $\sim 3600 \mu \mathrm{m}^{2}$ on $0.1 \mu \mathrm{g} / \mathrm{cm}^{2}$ substrates to $\sim 5000 \mu \mathrm{m}^{2}$ on $1.0 \mu \mathrm{g} / \mathrm{cm}^{2}$ and then increased only modestly to $\sim 5500 \mu \mathrm{m}^{2}$ on $10 \mu \mathrm{g} / \mathrm{cm}^{2}$ substrates (Fig. 2b). When we treated cells with a low dose of LPA $(1 \mu \mathrm{M}$ for $10 \mathrm{~min}$.), we found that spreading area remained constant compared to untreated controls on the 0.1 and $1 \mu \mathrm{g} / \mathrm{cm}^{2}$ substrates and fell modestly on the $10 \mu \mathrm{g} / \mathrm{cm}^{2}$ substrates (red bar, ${ }^{*} p<0.05$ ) (Fig. 2a, bottom panel; 
(a)

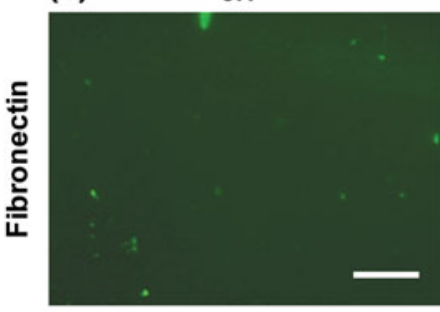

(b)
Fibronectin Density $\left(\mu \mathrm{g} / \mathrm{cm}^{2}\right)$

1.0

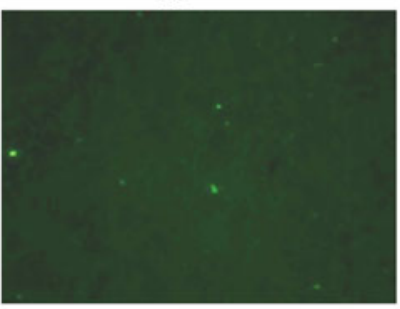

(c)
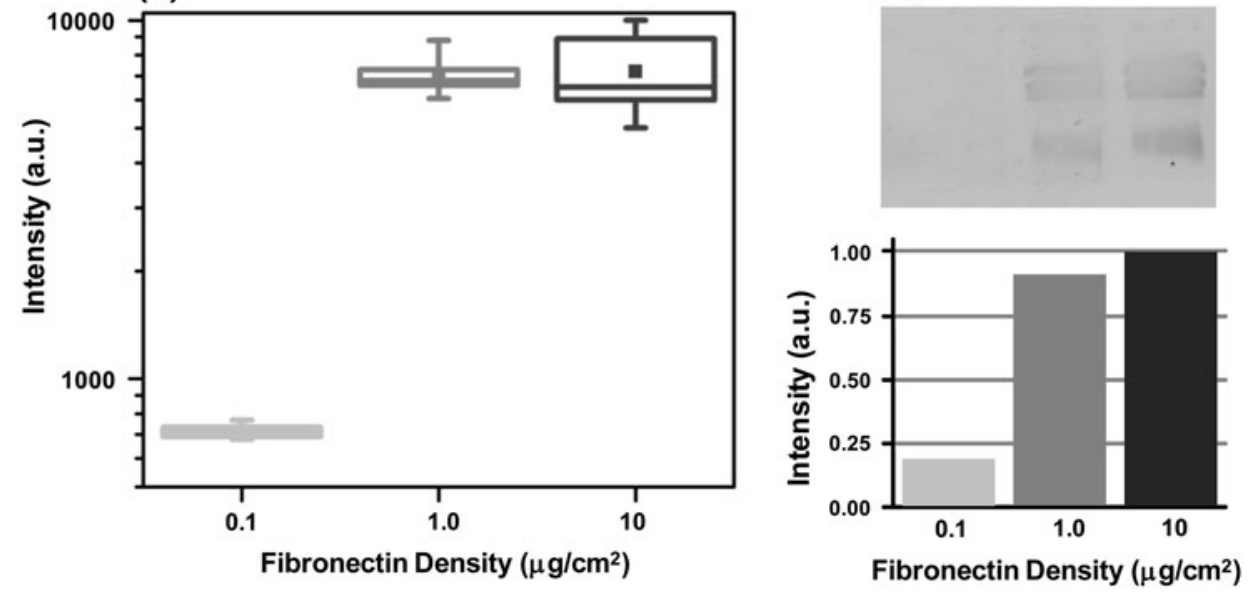

FIGURE 1. Quantification of surface adsorption of fibronectin (FN) at different densities. (a) Representative images of glass coverslips coated with FN at theoretical densities of $0.1,1.0$, and $10.0 \mu \mathrm{g} / \mathrm{cm}^{2}$, respectively, and probed with FN antibody (scale bar $=20 \mu \mathrm{m}$ ). (b) Intensity analysis of surface-adsorbed FN at different theoretical densities. Box-whisker plots of median intensities (10 random positions per condition) at different theoretical densities. (c) Quantification of surface-adsorbed FN by Western blot. FN adsorbed to glass coverslips at different densities was extracted and run on a blot to determine the amount of attached FN. Quantification of the band intensities by densitometry revealed a saturating trend with a fivefold increase in surfaceadsorbed FN between 0.1 and $1.0 \mu \mathrm{g} / \mathrm{cm}^{2}$.

Fig. 2b, right side). Under these conditions, then, LPA stimulates Rho GTPase but not to such an extent as to produce dramatic cell contraction.

\section{Fibronectin Density-Dependent De-adhesion Dynamics Under Serum-Free Conditions}

Having established conditions under which we could systematically vary matrix density and cell spreading, we next measured the kinetics of cell retraction following trypsin-mediated de-adhesion as described in our earlier study. ${ }^{21}$ Briefly, trypsin de-adhesion involves severing cell-matrix adhesive contacts with trypsin and following the cellular retraction that precedes cell detachment. During this process, projected cell area reduces from an initial area $A_{i}$ to a final area $A_{f}$ (Fig. $3 \mathrm{a}$ ). We then quantify retraction kinetics by tracking the average normalized cell-substrate contact area $\overline{A(t)}$ as a function of time. In the absence of LPA, retraction kinetics was largely independent of ECM coating density, with all cells fully retracting within $40 \mathrm{~s}$. In the presence of LPA, retraction kinetics remained independent of coating density but was greatly accelerated over untreated controls, with cells detaching within $20 \mathrm{~s}$ (Fig. 3b). The detachment response of the cells under all conditions was sigmoidal in nature, enabling us to fit each of these curves to a Boltzmann equation, which yielded two characteristic time constants, $\tau_{1}$ and $\tau_{2}$ (Fig. $3 \mathrm{c}$ ). Whereas $\tau_{1} \sim 8 \mathrm{~s}$ for control cells, $\tau_{1} \sim 4 \mathrm{~s}$ for LPAtreated cells, which represents a statistically significant (red bars, $* p<0.05$ ) difference of $50 \%$. Similarly, LPA treatment led to a statistically significant (red bars, $\left.{ }^{*} p<0.05\right) 45 \%$ reduction in $\tau_{2}(\sim 3 \mathrm{~s}$ and $\sim 1.65 \mathrm{~s}$ for control and LPA-treated cells, respectively). Taken together, these results demonstrate that under serumfree conditions, de-adhesion time constants are independent of fibronectin density but highly sensitive to contractile stimulation.

\section{Retraction Dynamics Correlate with Cytoskeletal} Architecture and Cell Mechanics on Fibronectin-Coated Substrates Under Serum-Free Conditions

The previous results illustrate that while spreading increases with fibronectin density under serum-free 
(a)
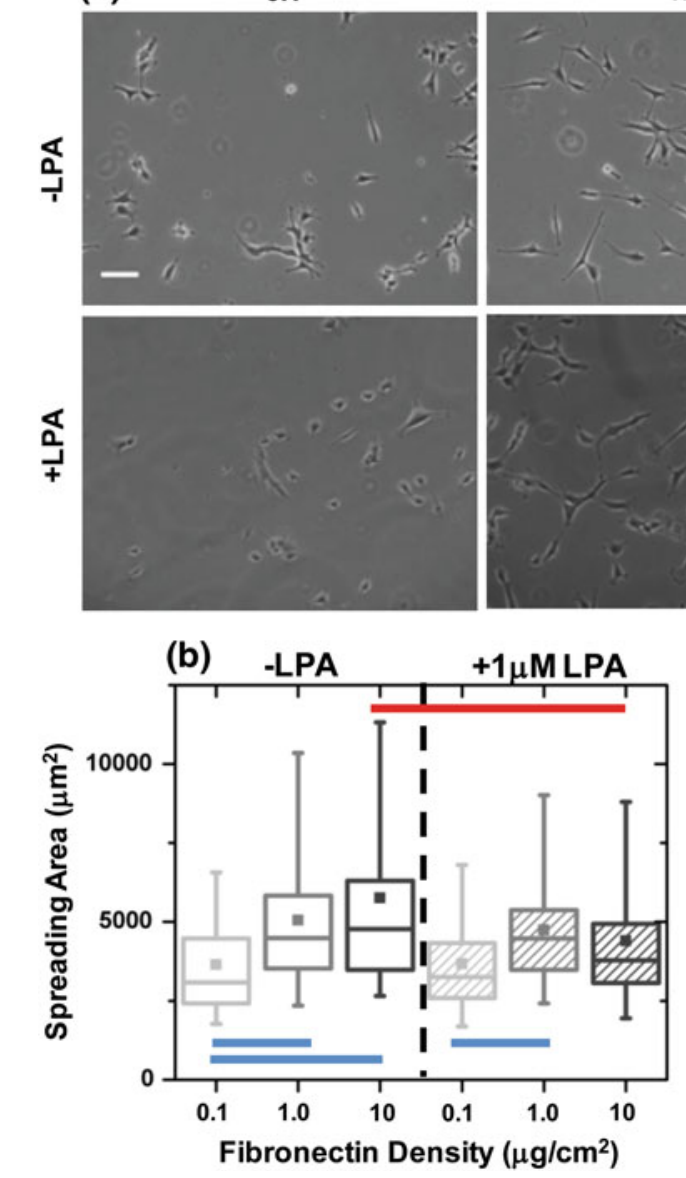

Fibronectin Density $\left(\mu \mathrm{g} / \mathrm{cm}^{2}\right)$ 1.0
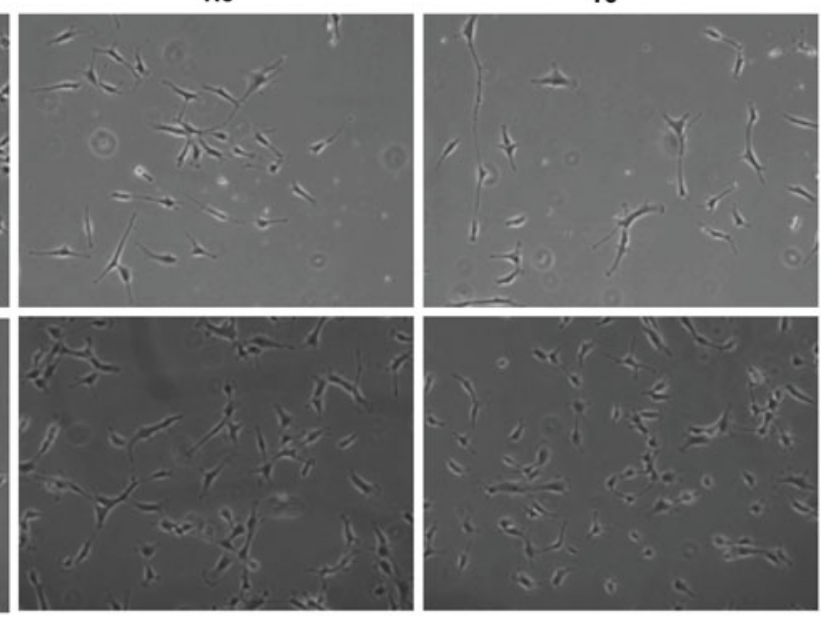

FIGURE 2. FN density-dependent spreading of U373 MG glioma cells under no-serum conditions. (a) U373 MG glioma cells were cultured on fibronectin-coated coverslips at different densities for $24 \mathrm{~h}$ under no-serum conditions. Representative images are shown of untreated cells (-LPA) and cells exposed to $1 \mu \mathrm{M}$ LPA for 10 min (+LPA). Scale bar = $10 \mu \mathrm{m}$. (b) For untreated cells, increase in fibronectin density led to increase in cell spreading in a statistically significant manner (blue bars, * $p<0.05)$. Contractile stimulation with LPA led to a significant reduction (red bar, ${ }^{*} p<0.05$ ) in cell spreading only on $10 \mu \mathrm{g} / \mathrm{cm}^{2}$ density substrates.

conditions, de-adhesion dynamics remain insensitive to density differences. Further, LPA treatment leads to faster de-adhesion dynamics despite only modestly altering cell shape. This suggests that cell contractility rather than adhesive ligand density is the principal determinant of cellular retraction kinetics. To address the possibility that matrix density might intrinsically contribute to cell contractility under these conditions, we investigated the effect of matrix density on cell stiffness and the assembly of the actomyosin cytoskeleton and cell-matrix adhesions. First, we used AFM nanoindentation to measure the elastic moduli of U373 MG glioma cells cultured on different density substrates with or without LPA treatment. AFM measurements confirmed that the distributions of cell stiffness for the three different densities were similar with mean stiffness of $\sim 3 \mathrm{kPa}$ (Fig. 4). LPA treatment did not alter cell stiffness for the 0.1 and $1.0 \mu \mathrm{g} / \mathrm{cm}^{2}$ densities, as evidenced by the substantial overlap in the box-whisker plots. However, on $10 \mu \mathrm{g} / \mathrm{cm}^{2}$ substrates, LPA-treated cells were significantly stiffer (red bar) than untreated controls with mean stiffness of $\sim 4 \mathrm{kPa}$. To gain additional microscale insight into these results, we used immunofluorescence imaging to visualize the localization of key elements of the actomyosin cytoskeleton and focal adhesion machinery. For all coating densities, both untreated control cells and LPA-treated cells exhibited prominent cortical actin bundles at cell margins but relatively few well-developed central stress fibers (Fig. 5). In the absence of LPA, vinculin and NMMII were diffusely distributed throughout the cytoplasm, with poor localization of NMMII to stress fibers and the absence of mature, well-defined focal adhesions. Addition of LPA increased localization of 
(a)

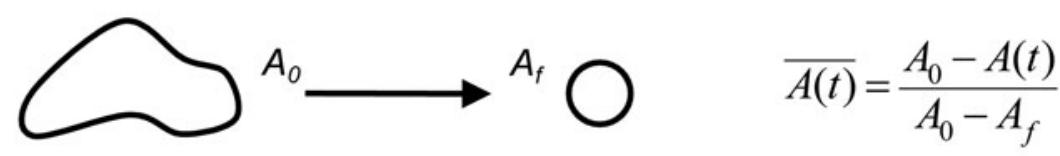

(b)

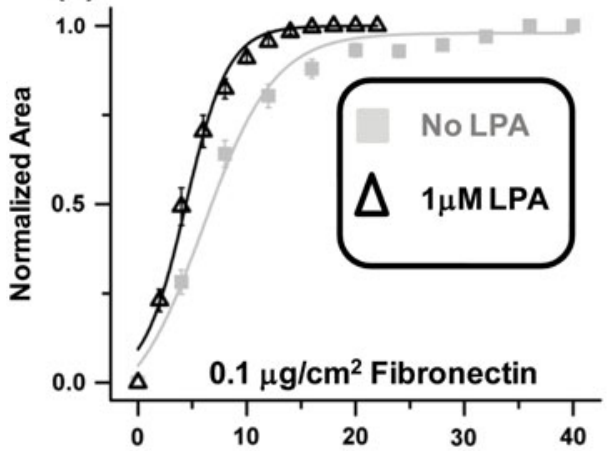

(c)
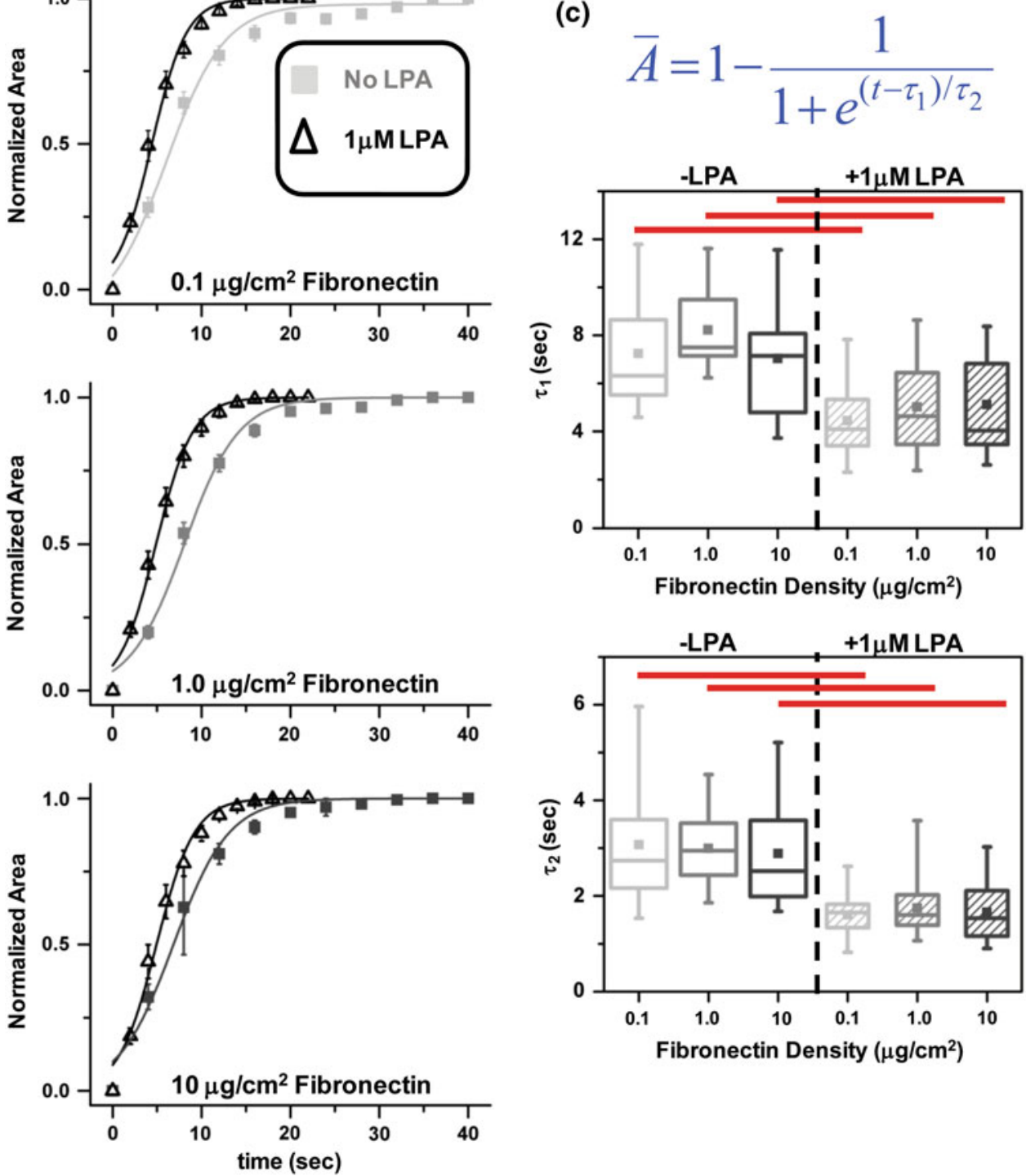

Fibronectin Density $\left(\mu \mathrm{g} / \mathrm{cm}^{2}\right)$

FIGURE 3. FN density-dependent de-adhesion dynamics of U373 MG glioma cells under no-serum conditions with and without LPA treatment. (a) Schematic of trypsin de-adhesion assay. Upon exposure to warm trypsin, the cell area reduces from an initial area $A_{i}$ to a final area $A_{f}$. Cells were imaged every $2 \mathrm{~s}$ until cells became rounded but remained attached. (b) Quantification of cell shape changes during de-adhesion on different FN-coated substrates under no-serum conditions. Plot of normalized cell area of untreated U373 MG glioma cells (-LPA) and cells exposed to $1 \mu \mathrm{M}$ LPA for 10 min (+LPA) cultured on different density FN-coated substrates. Irrespective of the FN density, LPA treatment caused faster de-adhesion. (c) Effect of FN density on time constants of retraction with and without LPA treatment. Under no-serum conditions, $\tau_{1}$ and $\tau_{2}$ were insensitive to changes in FN coating density. However, differences in $\tau_{1}$ and $\tau_{2}$ were statistically significant for LPA treatment (red bars, ${ }^{*} p<0.05$ ) across all the densities.

pMLC to stress fibers and membrane ruffles, even at coating densities $<10 \mu \mathrm{g} / \mathrm{cm}^{2}$ (white arrows). Overall, then, these data suggest that matrix density does not intrinsically alter contractile mechanics under these conditions and that LPA primes cell contractility through mobilization of NMMII to stress fibers and focal adhesions.

\section{Effect of Serum on Density-Dependent Spreading,} De-adhesion Dynamics and Cortical Mechanics on Fibronectin-Coated Substrates

Our results above establish that under serum-free, LPA-free conditions de-adhesion dynamics and cortical mechanics are independent of ECM density. While 
these studies enable us to work with well-defined ECM coatings that minimize ECM contributions from the medium, serum contains multiple components that are expected to influence cellular structure and organization, as evidenced by the fact that serum starvation induces a spindle-like morphology with poor formation of stress fibers and focal adhesions. Moreover, media formulations for many continuous cell lines contain high quantities of serum, and it would be valuable to know if these results also hold in serumrich medium. Therefore, we repeated all of the above experiments in culture medium containing $10 \%$ serum, standard growth conditions for U373MG glioma cells. In the presence of serum but in the absence of additional LPA, we observed consistent projected spreading areas $\left(\sim 5000 \mu \mathrm{m}^{2}\right)$ across all fibronectin densities (Fig. 6a). As in the serum-free case, LPA reduced cell spreading only on the $10 \mu \mathrm{g} / \mathrm{cm}^{2}$ substrates (red bar, $* p<0.05$ ). Intriguingly, in the presence of serum, cortical stiffness was found to be mildly responsive to changes in fibronectin density. The mean cortical stiffness of glioma cells increased significantly (blue bars, $* p<0.05$ ) from $\sim 2.75 \mathrm{kPa}$ on $1 \mu \mathrm{g} / \mathrm{cm}^{2}$ substrates to $\sim 4 \mathrm{kPa}$ on $1 \mu \mathrm{g} / \mathrm{cm}^{2}$ and $10 \mu \mathrm{g} / \mathrm{cm}^{2}$ substrates (Fig. 6b). This is presumably due to the presence of contractile agonists in serum that require threshold levels of integrin engagement for full activity. LPA treatment brought cells to a uniform stiffness value $(\sim 3.5 \mathrm{kPa})$ at all matrix densities.

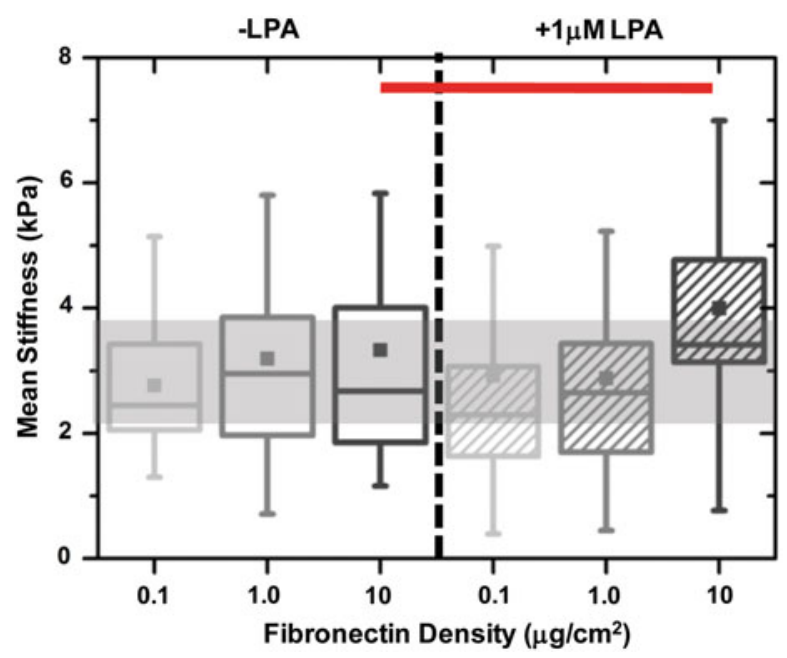

FIGURE 4. Effect of LPA on cortical stiffness of U373 MG cells cultured on different density FN substrates under no-serum conditions. Box-whisker plots of single cell stiffness of U373 MG glioma cells cultured on different density FN substrates with or without LPA treatment. Cell stiffness remained unchanged across all densities and upon LPA treatment on 0.1 and $1.0 \mu \mathrm{g} / \mathrm{cm}^{2}$ substrates. However, on $10.0 \mu \mathrm{g} / \mathrm{cm}^{2}$ substrates, LPA treatment led to a significant (red bar) increase in cell stiffness.
To determine whether these stiffness differences are also detectable in cellular retraction kinetics, we repeated our de-adhesion experiments in the presence of serum. Indeed, we observed density-dependent de-adhesion dynamics in the presence of serum with $\tau_{1} \sim 21 \mathrm{~s}, 17 \mathrm{~s}$, and $10 \mathrm{~s}$, respectively, for $0.1,1.0$, and $10 \mu \mathrm{g} / \mathrm{cm}^{2}$ substrates (Fig. 6c). The corresponding values for $\tau_{2}$ for the different densities were $6 \mathrm{~s}, 4.1 \mathrm{~s}$, and $3.7 \mathrm{~s}$, respectively. In LPA-treated cells, de-adhesion was further accelerated $\left(\tau_{1} \sim 7-8 \mathrm{~s}\right.$ and $\tau_{2} \sim 2 \mathrm{~s}$, respectively) and led to $\sim 70 \%$ reduction in both $\tau_{1}$ and $\tau_{2}$ on $0.1 \mu \mathrm{g} / \mathrm{cm}^{2}$ substrates. Further, as in the serumfree case, LPA treatment abolished density-dependent differences in de-adhesion dynamics. Taken together, these results demonstrate that in the presence of serum, while spreading responses become insensitive to changes in fibronectin density, de-adhesion dynamics and cortical mechanics are modulated in a density-dependent manner.

\section{DISCUSSION}

In this study we sought to separate contributions of cell-ECM adhesion and cytoskeletal prestress to cellular retraction kinetics following chemical detachment from the substrate. We have shown that in serum-free medium, increasing ECM coating density increases projected spreading area while only modestly affecting retraction kinetics. Conversely, stimulation of Rho GTPase-dependent contractility with LPA substantially accelerates retraction (i.e., reduces the time constant of retraction) regardless of the ECM coating density. When serum is re-introduced into the medium, the time constants of retraction fall with increasing ECM density; however, when LPA is added to further stimulate contractility, the time constants flatten to a constant, minimal value for all coating densities. Together, these data are consistent with a model in which cytoskeletal prestress dominates the total degree of cell-ECM adhesive engagement in determining retraction kinetics.

Importantly, these results neither demonstrate nor imply that the details of the cell-ECM adhesive interface bear no influence on retraction kinetics. Indeed, when we include serum in the medium, we find that the time constant of retraction rises significantly either in the presence or in the absence of LPA (Fig. 3c vs. Fig. 6c), with a moderate increase in cell stiffness by AFM (Fig. 4 vs. Fig 6b). Serum contains very high concentrations of fibronectin, albumin, and $\mathrm{IgG}$, which rapidly adsorb to culture surfaces and radically alter their surface chemistry. ${ }^{2,26}$ Serum also contains factors that strongly stimulate cellular synthesis of fibronectin and other matrix components, ${ }^{16}$ and 

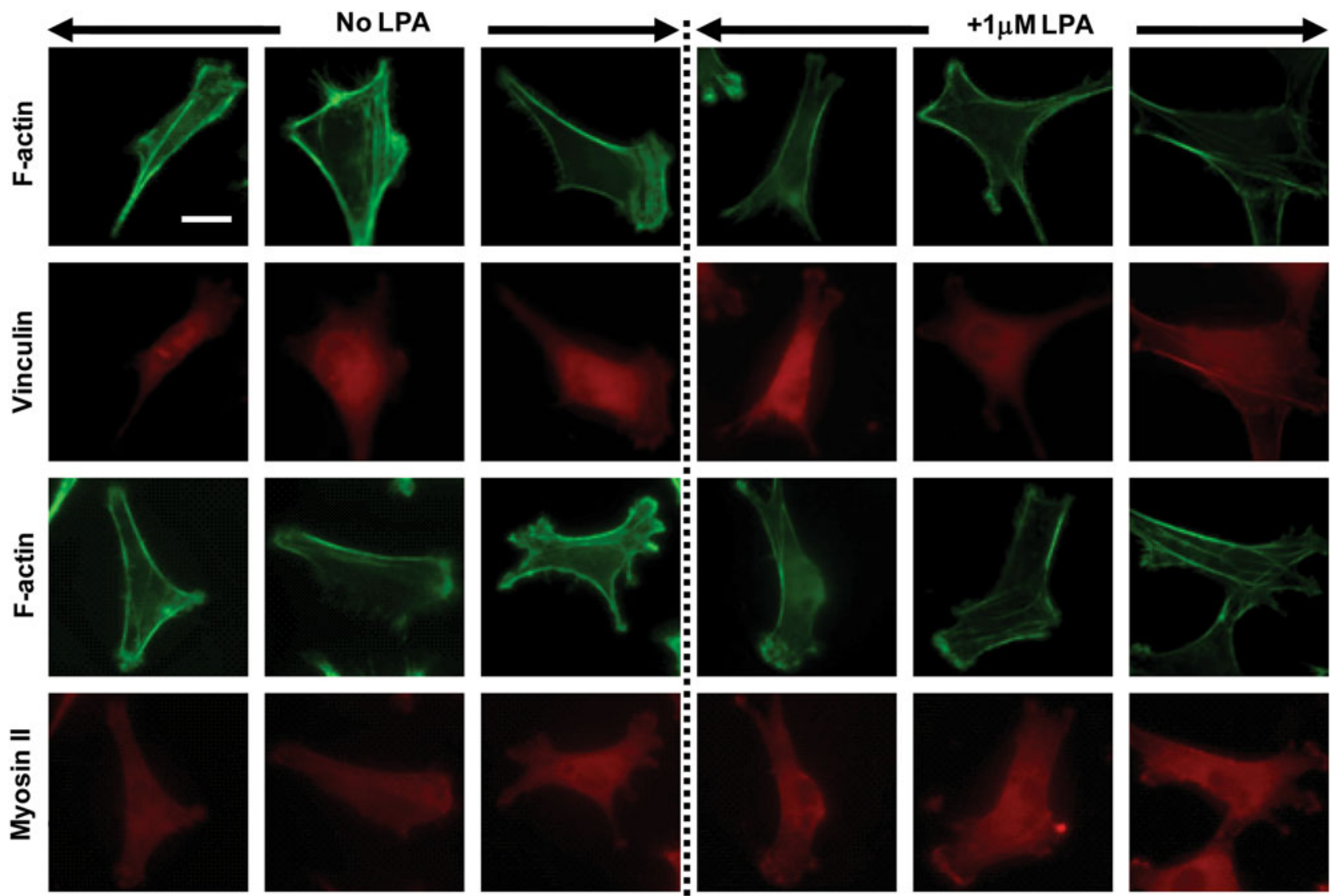

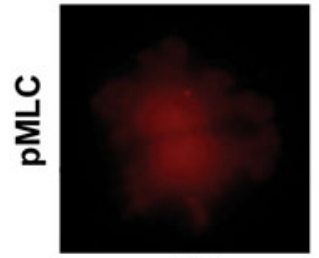

0.1

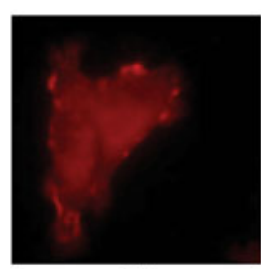

1.0

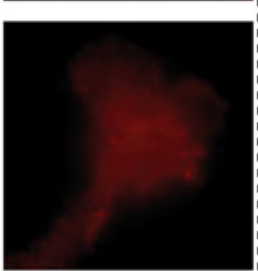

10

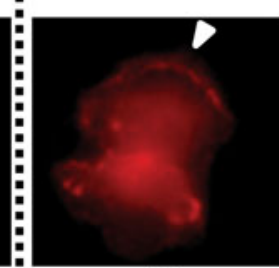

0.1

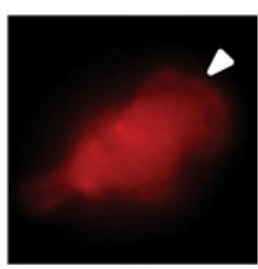

1.0

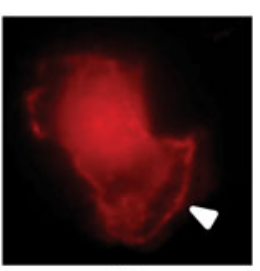

10

Fibronectin Density $\left(\mu \mathrm{g} / \mathrm{cm}^{2}\right)$

FIGURE 5. Cytoskeletal architecture, focal adhesions, NMMII, and pMLC localization. U373 MG cells cultured overnight in serumfree media on FN-coated substrates of different densities were treated with $1 \mu \mathrm{M}$ LPA for 10 min and immunostained for F-actin, vinculin, NMMII, and pMLC, respectively. Under serum-free conditions, glioma cells exhibit a predominantly peripheral actin staining with few central stress fibers. Vinculin staining at focal adhesions and NMMII were diffused both in controls and in LPAtreated cells across all the different densities. pMLC was mostly diffusely distributed in untreated cells, but sometimes localized to peripheral membrane ruffles in LPA-treated cells (white arrows). Scale bar $=20 \mu \mathrm{m}$.

adsorption of these factors can in turn alter the expression and activation of integrins and other adhesion receptors on the cell surface that are critical to mechanochemical feedback. The presence of these additional factors may require substantial time to fully disassemble and may even have varying degrees of trypsin sensitivity, which would affect retraction kinetics in unpredictable ways. LPA-induced adhesion maturation, as evidenced by pMLC recruitment, might also increase affinity per adhesion without changing spreading area and, therefore, require additional time for adhesion disassembly. The notion that adhesion disassembly may contribute to retraction measurements is also apparent from our earlier report, ${ }^{21}$ in which we showed that the speed of retraction increases with increasing activity (concentration) of trypsin. It is also potentially conceivable that differences in trypsininduced signaling across conditions could contribute to differences in measured retraction kinetics; however, we suspect this is unlikely given the extremely short time scale (tens of seconds) over which the measurements are made. All of that said, we do find that in the presence of serum, LPA stimulation of contractility still accelerates retraction kinetics, suggesting that even 

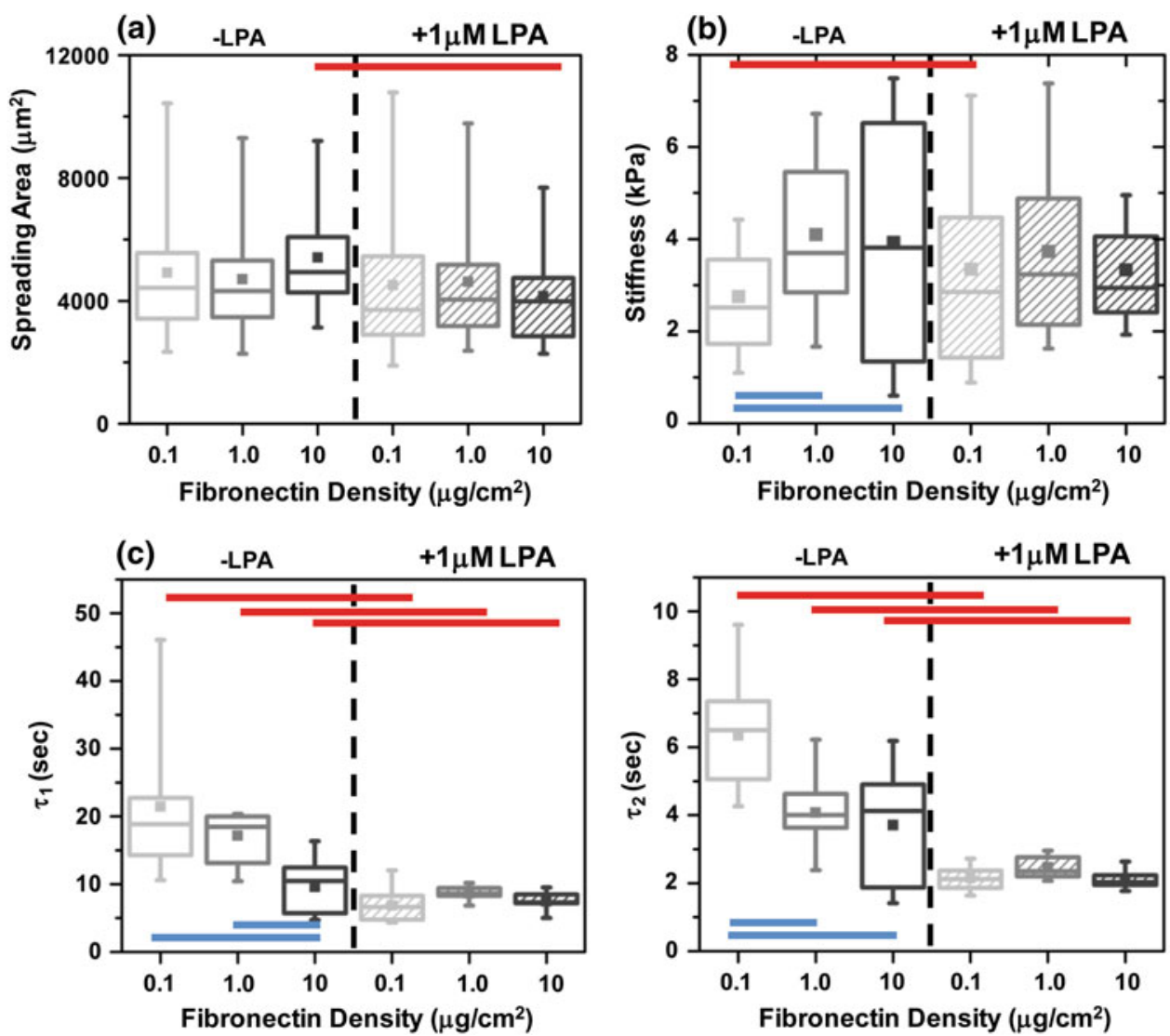

FIGURE 6. Effect of serum on the spreading, cortical stiffness, and de-adhesion dynamics of U373 MG cells cultured on different density FN substrates with and without LPA. (a) Under regular serum conditions, spreading remained unaltered on different FN-coated substrates. LPA treatment led to a reduction in spreading area with statistically significant differences observed only for the $10 \mu \mathrm{g} / \mathrm{cm}^{2}$ case (red bar, * $p<0.05$ ). (b) Cortical stiffness of U373 MG glioma cells cultured in the presence of serum on different density FN-coated substrates in the presence or in the absence of LPA. For untreated cells, increase in FN density led to cell stiffening from $\sim 2.85 \mathrm{kPa}$ measured on $0.1 \mu \mathrm{g} / \mathrm{cm}^{2}$ density substrates to $\sim 4 \mathrm{kPa}$ measured on 1 and $10 \mu \mathrm{g} / \mathrm{cm}^{2} \mathrm{density}$ substrates. LPA treatment eliminated these density-dependent differences in cell stiffness. (c) Effect of serum on FN density-dependent time constants of retraction with and without LPA treatment. For untreated cells cultured in serum-supplemented media, time constants exhibited an inverse density-dependent behavior with slowest retraction on $0.1 \mu \mathrm{g} / \mathrm{cm}^{2}$ substrates and fastest retraction on $10 \mu \mathrm{g} / \mathrm{cm}^{2}$ substrates. LPA treatment abolished this density-dependent behavior but led to even faster de-adhesion (red bars, * $p<0.05)$.

under "saturating" ECM ligand densities, modulations in contractility continue to measurably govern deadhesion kinetics.

Somewhat surprisingly, we find that under serumfree conditions, AFM measurements of cortical stiffness are relatively insensitive to either ECM coating density or addition of LPA (except at the highest ECM density) and that addition of serum does not dramatically increase the range of cortical stiffnesses (Figs. 4 and $5 b$ ). While the finding that addition of serum per se does not drastically alter cell stiffness may seem somewhat counterintuitive given the well-described ability of serum to induce stress fiber formation and focal adhesion maturation, ${ }^{19}$ this result is consistent with a previous set of observations with fibroblasts. ${ }^{18}$ In that study, cell stiffness by AFM did not change significantly in the setting of serum starvation, which the authors attributed to compensatory compaction of intermediate filaments around the nucleus. In fact, Hemmer et al. ${ }^{9}$ reported that serum starvation increases the AFM-measured stiffness of vascular smooth muscle cells, which they suggested may be secondary to serum-induced changes in shape and spreading area. The equivocal effects of serum on cellular elasticity further validate our decision to establish proof-of-principle in serum-free medium and stimulate contractility with LPA, which is a chemically homogeneous stimulus that induces contractility via a single, defined intracellular target.

Remarkably, we observe that retraction dynamics measurements are frequently much more sensitive than AFM indentation measurements to LPA-induced stimulation of contractility. This is despite the fact that many studies support a close correlation between cellular contractility (or prestress) and cortical stiffness and have shown that manipulation of NMMII activity 
leads to predictable changes in cell stiffness. ${ }^{21,23,29} \mathrm{We}$ hypothesize that the higher apparent sensitivity of the de-adhesion assay arises from three sources. First, de-adhesion measurements represent an integrative measure of the contractile state of the entire cell, whereas AFM measurements are localized to nano- to microscale regions of the cell surface. Second, the high throughput of the de-adhesion measurements enables significantly larger sample sizes and statistical power, which in turn may more easily elicit differences between conditions. Third, it is possible that LPA may induce stiffening of basal stress fibers, which presumably make the largest contributions to the retraction dynamics, to a much greater degree or on a faster time scale than it induces stiffening of the cortical actomyosin elements probed by AFM. An alternative explanation is that AFM may not be sufficiently sensitive to detect changes in contractile forces exerted at the cell base, and so in the future it would be valuable to repeat these experiments with direct measurements of traction forces, including traction force microscopy. Evidence that such an approach could yield results consistent with ours was recently provided by Califano et al., ${ }^{1}$ who demonstrated that traction forces exerted by endothelial cells depend strongly on substrate stiffness, but not on ligand density.

Several open questions remain. First, whereas we performed all of our studies with fibronectin, these studies clearly require repetition with matrix components that assemble into higher-order, micron-scale structures, such as collagen I. Collagen fiber assembly and dimensions depend strongly on collagen concentration, ${ }^{25}$ and it is conceivable that as matrix features begin to approach the size of individual cells, fiber microarchitecture could influence retraction kinetics in ways that are both related to and independent of effects on cytoskeletal prestress. Second, it would be valuable to develop a more quantitative relationship between prestress and the time constant of retraction, with an eye toward creating a "standard curve" that could permit deduction of one parameter from the other. The dose-response performance of LPA may not be sufficiently high to permit this level of control, but other perturbations might. Third, our studies make no attempt to control cell geometry, which could influence both prestress and adhesive area. It would, therefore, be informative to repeat these studies with single cells under constant ECM protein density but variable geometry (e.g., by micropatterning single cells) and ask whether de-adhesion kinetics are affected by spreading area, aspect ratio, and other parameters.

In summary, our data show that the retraction kinetics of adherent cells following trypsin-mediated of detachment are more sensitive to cytoskeletal prestress than to the extent of cell-matrix adhesion. These studies support further exploration of these measurements as a low-resolution, high-throughput approach for screening mechanical properties of adherent cells, and additional effort should help assess the generalizability of these findings across cell types, matrix formulations, and media conditions.

\section{ACKNOWLEDGMENTS}

WPN was supported by a UC Berkeley Graduate Fellowship. SK gratefully acknowledges the support of the Arnold and Mabel Beckman Young Investigator Award, the NSF (CMMI 0727420), a PECASE award from the Army Research Office (W911NF-09-1-0507), an NIH Physical Sciences Oncology Center Grant (1U54CA143836), and an NIH Director's New Innovator Award (1DP2OD004213), a part of the NIH Roadmap for Medical Research.

\section{OPEN ACCESS}

This article is distributed under the terms of the Creative Commons Attribution Noncommercial License which permits any noncommercial use, distribution, and reproduction in any medium, provided the original author(s) and source are credited.

\section{REFERENCES}

${ }^{1}$ Califano, J., and C. Reinhart-King. Substrate stiffness and cell area predict cellular traction stresses in single cells and cells in contact. Cel. Mol. Bioeng. 3(1):68-75, 2010.

${ }^{2}$ Dekker, A., K. Reitsma, T. Beugeling, A. Bantjes, J. Feijen, and W. G. van Aken. Adhesion of endothelial cells and adsorption of serum proteins on gas plasma-treated polytetrafluoroethylene. Biomaterials 12(2):130-138, 1991.

${ }^{3}$ Discher, D., C. Dong, J. J. Fredberg, F. Guilak, D. Ingber, P. Janmey, R. D. Kamm, G. W. Schmid-Schonbein, and S. Weinbaum. Biomechanics: cell research and applications for the next decade. Ann. Biomed. Eng. 37(5):847-859, 2009. ${ }^{4}$ Engel, A., and H. E. Gaub. Structure and mechanics of membrane proteins. Annu. Rev. Biochem. 77:127-148, 2008. ${ }^{5}$ Engler, A. J., F. Rehfeldt, S. Sen, and D. E. Discher. Microtissue elasticity: measurements by atomic force microscopy and its influence on cell differentiation. Methods Cell Biol. 83:521-545, 2007.

${ }^{6}$ Engler, A. J., P. O. Humbert, B. Wehrle-Haller, and V. M. Weaver. Multiscale modeling of form and function. Science 324(5924):208-212, 2009.

${ }^{7}$ Guck, J., R. Ananthakrishnan, H. Mahmood, T. J. Moon, C. C. Cunningham, and J. Kas. The optical stretcher: a novel laser tool to micromanipulate cells. Biophys. J. 81(2):767-784, 2001.

${ }^{8}$ Hale, C. M., S. X. Sun, and D. Wirtz. Resolving the role of actoymyosin contractility in cell microrheology. PLoS ONE 4(9):e7054, 2009. 
${ }^{9}$ Hemmer, J. D., D. Dean, A. Vertegel, E. Langan, III, and M. LaBerge. Effects of serum deprivation on the mechanical properties of adherent vascular smooth muscle cells. Proc. Inst. Mech. Eng. H 222(5):761-772, 2008.

${ }^{10}$ Hochmuth, R. M. Micropipette aspiration of living cells. J. Biomech. 33(1):15-22, 2000.

${ }^{11}$ Ignotz, R., and J. Massagué. Transforming growth factorbeta stimulates the expression of fibronectin and collagen and their incorporation into the extracellular matrix. J. Biol. Chem. 261(9):4337-4345, 1986.

${ }^{12}$ Lincoln, B., F. Wottawah, S. Schinkinger, S. Ebert, and J. Guck. High-throughput rheological measurements with an optical stretcher. Methods Cell Biol. 83:397-423, 2007.

${ }^{13}$ Mills, G. B., and W. H. Moolenaar. The emerging role of lysophosphatidic acid in cancer. Nat. Rev. Cancer 3(8): 582-591, 2003.

${ }^{14}$ Mills, J. P., L. Qie, M. Dao, C. T. Lim, and S. Suresh. Nonlinear elastic and viscoelastic deformation of the human red blood cell with optical tweezers. Mech. Chem. Biosyst. 1(3):169-180, 2004.

${ }^{15}$ Musielak, M. Red blood cell-deformability measurement: review of techniques. Clin. Hemorheol. Microcirc. 42(1): 47-64, 2009.

${ }^{16}$ Namura, T., S. Arai, K. Okawa, A. Koike, S. Yamada, N. Saita, A. Nagae, H. Itoh, M. Totani, S. Uemoto, and M. Ikemoto. Identification of serum proteins that bind with S100A8, S100A9 and S100A8/A9: clinical significance of using proteins for monitoring the postoperative condition of liver recipients. Clin. Chim. Acta 411(21-22):1766-1773, 2010.

${ }^{17}$ Paszek, M. J., N. Zahir, K. R. Johnson, J. N. Lakins, G. I. Rozenberg, A. Gefen, C. A. Reinhart-King, S. S. Margulies, M. Dembo, D. Boettiger, D. A. Hammer, and V. M. Weaver. Tensional homeostasis and the malignant phenotype. Cancer Cell 8(3):241-254, 2005.

${ }^{18}$ Pelling, A. E., D. W. Dawson, D. M. Carreon, J. J. Christiansen, R. R. Shen, M. A. Teitell, and J. K. Gimzewski. Distinct contributions of microtubule subtypes to cell membrane shape and stability. Nanomedicine 3(1):43-52, 2007.

${ }^{19}$ Ridley, A. J., and A. Hall. The small GTP-binding protein rho regulates the assembly of focal adhesions and actin stress fibers in response to growth factors. Cell 70(3): 389-399, 1992.

${ }^{20}$ Rotsch, C., K. Jacobson, and M. Radmacher. Dimensional and mechanical dynamics of active and stable edges in motile fibroblasts investigated by using atomic force microscopy. Proc. Natl. Acad. Sci. USA 96(3):921-926, 1999.

${ }^{21}$ Sen, S., and S. Kumar. Cell-matrix de-adhesion dynamics reflect contractile mechanics. Cel. Mol. Bioeng. 2(2):218230, 2009.

${ }^{22}$ Sen, S., and S. Kumar. Combining mechanical and optical approaches to dissect cellular mechanobiology. J. Biomech. 43(1):45-54, 2010.

${ }^{23}$ Sen, S., and S. Kumar. Isoform-specific contributions of $\alpha$-actinin to glioma cell mechanobiology. PLoS ONE 4(12): e8427, 2009.

${ }^{24}$ Suresh, S. Biomechanics and biophysics of cancer cells. Acta Biomater. 3(4):413-438, 2007.

${ }^{25}$ Ulrich, T. A., A. Jain, K. Tanner, J. L. MacKay, and S. Kumar. Probing cellular mechanobiology in threedimensional culture with collagen-agarose matrices. Biomaterials 31(7):1875-1884, 2010.

${ }^{26}$ van Wachem, P. B., C. M. Vreriks, T. Beugeling, J. Feijen, A. Bantjes, J. P. Detmers, and W. G. van Aken. The influence of protein adsorption on interactions of cultured human endothelial cells with polymers. J. Biomed. Mater. Res. 21(6):701-718, 1987.

${ }^{27}$ Vogel, V., and M. P. Sheetz. Cell fate regulation by coupling mechanical cycles to biochemical signaling pathways. Curr. Opin. Cell Biol. 21(1):38-46, 2009.

${ }^{28}$ Wang, J. H., and J. S. Lin. Cell traction force and measurement methods. Biomech. Model. Mechanobiol. 6(6): 361-371, 2007.

${ }^{29}$ Wang, N., I. M. Tolic-Norrelykke, J. Chen, S. M. Mijailovich, J. P. Butler, J. J. Fredberg, and D. Stamenovic. Cell prestress. I. Stiffness and prestress are closely associated in adherent contractile cells. Am. J. Physiol. Cell Physiol. 282(3):C606C616, 2002.

${ }^{30}$ Wildt, B., D. Wirtz, and P. C. Searson. Programmed subcellular release for studying the dynamics of cell detachment. Nat. Methods 6(3):211-213, 2009.

${ }^{31}$ Wirtz, D. Particle-tracking microrheology of living cells: principles and applications. Annu. Rev. Biophys. 38:301326, 2009.

${ }^{32}$ Yao, A., M. Tassieri, M. Padgett, and J. Cooper. Microrheology with optical tweezers. Lab Chip 9(17):2568-2575, 2009.

${ }^{33}$ Zhang, H., and K. K. Liu. Optical tweezers for single cells. J. R. Soc. Interface 5(24):671-690, 2008. 